\title{
COMPETITIVE EQUILIBRIA IN DECENTRALIZED MATCHING WITH INCOMPLETE INFORMATION
}

\author{
ALP E. ATAKAN
}

\begin{abstract}
.
This paper shows that all perfect Bayesian equilibria of a decentralized dynamic matching market with two-sided incomplete information of independent private values variety converge to competitive equilibria. Each buyer wants to purchase a bundle of heterogeneous, indivisible goods and each seller owns one unit of a heterogeneous indivisible good (as in Kelso and Crawford (1982) or Gul and Stacchetti (1999)). Buyer preferences and endowments as well as seller costs are private information. Agents engage in costly search and meet randomly. The terms of trade are determined through bilateral bargaining between buyers and sellers. The paper considers a market in steady state. It is shown that as frictions, i.e., discounting and fixed costs of search become small, all equilibria of the market game converge to perfectly competitive equilibria.
\end{abstract}

Keywords: Bargaining, Search, Matching

JEL Classification Numbers: C73, C78, D83.

\section{INTRODUCTION}

This paper shows that all equilibria of a dynamic matching and bargaining game with twosided incomplete information of the independent private values variety converge to competitive equilibria. In the model each buyer aims to purchase a bundle of heterogeneous, indivisible objects and each seller owns one unit of a heterogeneous indivisible good (as in Kelso and Crawford (1982) or Gul and Stacchetti (1999)). Buyer preferences and endowments as well as seller costs are private information. Agents engage in costly search and meet randomly. The terms of trade are determined through bilateral bargaining under incomplete information between buyers and sellers. The paper considers a market in steady state and shows that as frictions, i.e., discounting and the fixed cost of search, become small for all agents, the market becomes perfectly competitive.

Convergence to a competitive equilibrium in a decentralized market for a homogeneous good is established by Gale (1987). This result is extended to the case of incomplete information about buyer preferences in a two-type model by Moreno and Wooders (2002) and two-sided incomplete information by Satterthwaite and Shneyerov (2006). In the models of DeFraja and Sakovics (2001), Serrano (2002) and Wolinsky (1990) convergence to a competitive equilibrium fails. The failure of convergence to competitive equilibrium is caused by the bilateral bargaining protocol in Serrano (2002), results from the inefficiency of aggregating common value information through bilateral 
meeting in Wolinsky (1990) and is due to the "clones" assumption in DeFraja and Sakovics (2001) (see Lauermann (2006) for a detailed discussion of these issues).

\section{The ModeL}

Buyers and sellers in the economy search for possible trading partners. Each seller owns one indivisible good for sale and each buyer wants to purchase a bundle of the indivisible goods offered for sale. The game progresses in discrete time and agents discount the future with a common discount factor $\delta$. In each period, an agent incurs a positive search cost $c$ and meets pairwise with a potential partner. Either the buyer or the seller is designated as the proposer. The probability that the buyer is designated as the proposer is $\beta \in(0,1)$. The proposer offers a direct mechanism and the responder chooses whether to participate in the mechanism. If the responder participates in the mechanism, then the agents report their types to the mechanism and the mechanism chooses the probability with which a trade occurs and specifies the transfers to be paid by the buyer to the seller. Sellers who trade permanently leave the market. Buyers remain in the economy until they have purchased all the goods that they want, then they leave the market and consume their bundle. Agents who fail to trade return to the searching population. Utility is transferable. If a buyer of type $b$ consumes bundle $G$, then she enjoys utility $h_{b G}$. A seller incurs cost $r_{s}$ when she sells her good. So trade between $b$ and sellers $s \in G$ creates total transferable utility $f_{b G}=h_{b G}-\sum_{s \in G} r_{s}$.

2.1. Population of Types and Private Information. Let $B$ and $S$ denote the finite sets of initial buyer and seller types and $I=B \cup S$ denotes the set of all initial types. A seller's type specifies the good she owns, $x_{s}$, and her reservation value (or cost) $r_{s}$. Let $X$ denote the set of goods potentially traded in the market, i.e, if $x_{s}$ is the good owned by seller $s$, then $X=\left\{x_{s}: s \in S\right\}$. A buyer's initial type specifies the buyer's utility function $h_{b}: \mathcal{P}(S) \rightarrow \mathbb{R}$, where $\mathcal{P}(S)$ denotes the set of all subsets of $S$. The utility function for any buyer $b$ satisfies:

(i) $h_{b \emptyset}=0$,

(ii) Monotonicity: If $G \supset A$, then $h_{b G} \geq h_{b A}$

(iii) Identity Independence: For any $s$ and $s^{\prime}$ with $x_{s}=x_{s^{\prime}}$ (i.e., sellers who own the same good), $h_{b G \cup\{s\}}=h_{b G \cup\left\{s^{\prime}\right\}}$ for all $G$.

Once in the market, a buyer's type changes after each trade and includes information on all trades that the agent has made and consequently the goods the buyer owns. So, refer to a buyer type by $b_{G}$, where $b \in B$ is the initial type, i.e., her utility function, and $G$ is the set of seller types with whom she has already traded. Consequently, the set of buyer types present in the market is $T=B \times \mathcal{P}(S)$.

In each period, a unit measure of each $b \in B$ and $s \in S$ are available to enter the market. Consequently, in each period a measure $|B|$ of buyers and measure $|S|$ of sellers potentially enter the market. Buyers and sellers, who do not enter the market in a given period, are assumed to have opted for an outside option and are thus not available for entry in any subsequent periods. 
Let $l=\left(l_{b 1}, \ldots, l_{b_{G}}, \ldots, l_{|B|_{\mid 2} S}, l_{s 1}, \ldots, l_{|S|}\right)$ denote the steady state measure of buyers and sellers in the market, i.e., $l \in \mathbb{R}_{+}^{|B| \times\left|2^{S}\right|+|S|}$. The steady state probability for any seller of meeting buyer $b$, or any buyer meeting a seller $s$ in a given period is

$$
p_{b}=\frac{l_{b}}{\max \left\{L_{B}, L_{S}\right\}} \quad \text { or } \quad p_{s}=\frac{l_{s}}{\max \left\{L_{B}, L_{S}\right\}}
$$

where $L_{B}=\sum_{b \in T} l_{b}$ and $L_{S}=\sum_{s \in S} l_{s}$. The total measure of buyer $b$ and seller $s$ pairs formed in a period is $l_{s} p_{b}=l_{b} p_{s}$ and the total measure of pairs formed is equal to $\min \left\{L_{B}, L_{S}\right\}$. The (sub) probability measures $p_{b}$ and $p_{s}$ (or type distributions) are commonly known by all agents.

The analysis here assumes independent private values. More precisely, when a buyer and seller make a trade, the payoff to each agent depends only on the terms of trade and their own private information but not on their trading partner's private information. Reference to this assumption, which is stated formally below, is omitted from the statements of the results presented since it is maintained throughout the paper.

Assumption: Independent Private Values. If buyer $b_{G}$ and seller $s$ meet, then the buyer observes, $x_{s}$, the good that seller $s$ has for sale, while $b_{G}$ and $r_{s}$ remain as private information.

Also, further assume that the agents know the distribution of types in the economy and that any agent's prior belief about his/her trading partner's type coincides with the distribution of types. This requirement is stronger than what is needed for showing convergence to a competitive equilibrium. As long as the support of any agent's prior belief coincides with the support of the steady state distribution, the convergence results will continue to hold.

2.2. Agent Behavior and Strategies. Let $\sigma_{i}$ denote a strategy for type $i$ and $\sigma=\left(\sigma_{i}\right)_{i \in I}$ a strategy profile. Assume that all agents use stationary time-invariant strategies $\left(\sigma^{t}=\sigma\right.$ for all $\left.t\right)$. At the start of each period, the strategy determines whether the agent remains in (or enters) the market and pays the cost $c$. Denote by $\sigma_{i}(i n)$ the probability that agent $i$ remains in (or enters) the market in any period. If $i$ is paired in the current period and is the proposer, then the strategy $\sigma_{i}$ returns a (direct) mechanism choice $\mu_{i}$. If agent $i$ is the responder, then the strategy specifies whether she accepts to participate in the mechanism. If the responder accepts to participate, then the two agents report types $k$ and $j$ to the mechanism and the mechanism chooses a probability of trade and the transfer to be paid by the buyer to the seller. The proposer can condition her mechanism offer and the responder can condition her type report on: the measure of agents in the economy $l$, other common knowledge parameters of the economy, and the observable characteristics of her partner for the period. The responder can also condition her report on the mechanism choice of the proposer. Without loss of generality, assume that the proposer offers an individually rational and (interim) incentive compatible mechanism and the responder always participates and reports her type truthfully. Consequently, a strategy for type $i$ is given by $\sigma_{i}=\left(\sigma_{i}(i n), \mu_{i}\right)$. 
The game played in each period involves a direct mechanism choice, that satisfies individual rationality and incentive compatibility constraints, by an informed proposer (principle) (see for example Myerson (1983) or Maskin and Tirole (1990)). However, the Lemma below shows that the private information of the proposer is unimportant. In fact, the proposer chooses the same mechanism she would have chosen even if her type was publicly know. In particular, since buyers and sellers are risk neutral, a take-it-or-leave-it offer is an optimal mechanism for the proposer (as in Riley and Zeckhauser (1983)).

Lemma 1. The equilibrium mechanism choices satisfy the following:

(i) If $\mu_{i}$ is the optimal (direct) mechanism choice for type $i$, when type $i$ is proposing and her type is known to the responder, then the direct mechanism $\mu=\left(\mu_{i}\right)$, i.e., the mechanism that uses $\mu_{i}$ when the proposer reports type $i$ to the mechanism, is an optimal mechanism choice for all $i$, when the proposer's type is private information.

(ii) A take-it-or-leave-it offer is optimal when the proposer's type is know, consequently a type specific take-it-or-leave-it offer is an optimal mechanism for any proposer.

(iii) If $s$ and $s^{\prime}$ own the same good and $r_{s} \geq r_{s}^{\prime}$ then the take-it-or-leave-it offer $t_{s} \geq t_{s}^{\prime}$ in any equilibrium.

In the case where each buyer only wants to purchase a single good, then a property analogous to item (iii) also holds for all buyers.

Let the match probability $m_{b s}$ ( or $m_{s b}$ ) denote the probability that $b$ and $s$ trade, given that the two are paired in the period and $b$ (or $s$ ) is chosen as the proposer. Also, let $t_{b s}$ (or $t_{s b}$ ) denote the transfer paid by the buyer to the seller, given that $b$ and $s$ are paired in the period, $b$ (or $s$ ) is chosen as the proposer and they trade. The reward function for a buyer $b$ (or seller $s$ ) proposing (responding) to seller $s$ (buyer $b$ ) is $\pi_{b}(\sigma, s)=-c-m_{b s} t_{b s}\left(\right.$ or $\pi_{s}(\sigma, b)=-c+m_{b s}\left(t_{b s}-r_{s}\right)$ ). If an agent has exited the market or has accepted a match in a prior period, then the agent is paired with 0 , and $\pi_{i}(\sigma, 0)=0$. Also, if the agent does not get paired in a period, then she/he is paired with herself and $\pi_{i}(\sigma, i)=-c$.

The expected future value at the start of a period for a seller equals the maximum of the value of remaining in the market and the value of leaving the market, i.e., $v_{s}=\max \left\{v_{s}(i n), 0\right\}$. The expected future value at the start of a period for a buyer equals the maximum of the value of remaining in the market and the value of leaving the market and consuming the bundle that they own, that is, $v_{b}=\max \left\{v_{b}(i n), h_{b G(b)}\right\}$ where for each $b \in T, G(b)$ denotes the set of seller types with whom type $b$ has already traded. The value of remaining in the economy, $v_{b}(i n)$, satisfies

$$
\begin{aligned}
v_{b}(i n)=-c+\sum_{s} p_{s} \beta m_{b s}\left(\delta v_{b \cup s}-\right. & \left.t_{b s}\right)+\sum_{s} p_{s}(1-\beta) m_{s b}\left(\delta v_{b \cup s}-t_{s b}\right) \\
+ & \left(1-\sum_{s} p_{s} \beta m_{b s}-\sum_{s} p_{s}(1-\beta) m_{s b}\right) \delta v_{b}
\end{aligned}
$$


where the notation $b \cup s$ (or $b \backslash s$ ) denotes a type $b^{\prime}$ with $h_{b}=h_{b^{\prime}}$ and $G\left(b^{\prime}\right)=G(b) \cup\{s\}$ (or $\left.G\left(b^{\prime}\right)=G(b) \backslash s\right)$. In words, buyer $b$ pays the search (sampling) cost $c$, then successfully makes a trade as the responder with seller $\mathrm{s}$ with probability $(1-\beta) p_{s} m_{s b}$; makes a trade when she proposes to buyer $s$ with probability $\beta p_{s} m_{b s}$; and does not trade in the period and receives her continuation value $\delta v_{b}$ with probability $\left(1-\sum_{s} p_{s} \beta m_{b s}-\sum_{s} p_{s}(1-\beta) m_{s b}\right)$. Continuation values are defined similarly for the sellers. Rearranging gives the following for buyer and seller values:

$$
\begin{aligned}
v_{s}(i n)=-c+\beta \sum_{b \in T} p_{b} m_{b s}( & \left.t_{b s}-r_{s}-\delta v_{s}\right) \\
+ & (1-\beta) \sum_{b \in T} p_{b} m_{s b}\left(t_{s b}-r_{s}-\delta v_{s}\right)+\delta v_{s}, \text { and } \\
v_{b}(i n)=-c+\beta \sum_{s} p_{s} m_{b s}( & \left.\delta v_{b \cup s}-\delta v_{b}-t_{b s}\right) \\
+ & (1-\beta) \sum_{s} p_{s} m_{s b} \delta\left(v_{b \cup s}-\delta v_{b}-t_{s b}\right)+\delta v_{b},
\end{aligned}
$$

for any $b$ and $s$.

2.3. Steady State. Assuming that the economy remains in steady state implies that the number of type $b$ buyers (or type $s$ sellers) entering the market in each period must equal the number of that type leaving the market. Consequently, the steady state measure of agents in the economy, $l$, satisfies the following steady state equations

$$
\begin{aligned}
& l_{b}\left(\sum_{s \in S} M_{b s}+\sigma_{b}(\text { out })\right)=\sigma_{b}(\text { in }) \\
& l_{s}\left(\sum_{b \in T} M_{s b}+\sigma_{s}(\text { out })\right)=\sigma_{s}(\text { in })
\end{aligned}
$$

for all types $b \in T$ with $b(G)=\emptyset$ and all sellers $s \in S$, where $M_{b s}=p_{s}\left(\beta m_{b s}+(1-\beta) m_{s b}\right)$ denotes the fraction of type $b$ buyers who are successfully matched with type $s$ sellers, $\sigma_{b}($ out $)=$ $\left(1-\sum_{s \in S} M_{b s}\right)\left(1-\sigma_{b}(i n)\right)$ denotes the fraction of type $b$ buyers, who failed to trade in the previous period, that choose to leave at start of the current period, and $\sigma_{b}(i n) \leq 1$ is the flow of new buyers into the market at the start of the period. Also,

$$
l_{b}\left(\sum_{s \in S} M_{b s}+\sigma_{b}(\text { out })\right)=\sigma_{b}(\text { in }) \sum_{s \in G(b)} l_{b \backslash s} M_{b \backslash s s}
$$

for $b \in T$ with $b(G) \neq \emptyset$, where $\sigma_{b}(i n) \sum_{s \in G(b)} l_{b \backslash s} M_{b \backslash s s}$ is the measure of newly created type $b$ buyers who remain in the market.

2.4. Equilibrium. A steady state search equilibrium is comprised of a mutually compatible strategy profile $\sigma$ and steady state measure $l$. That is to say, the measure $l$ satisfies the steady state equations, given that agents use strategy profile $\sigma$ and, the strategy profile $\sigma$ comprises a Perfect Bayesian Equilibrium for the market game, given that the steady state measure of agent is $l$.

2.5. The Competitive Benchmark. The competitive equilibrium benchmark considered here is a "flow" equilibrium as in Gale (1987) or Satterthwaite and Shneyerov (2006), generalized to 
accommodate heterogenous goods and multi-unit demand. In each period, flow supply is the measure of sellers of a particular good entering the market and flow demand is the measure of agents willing to purchase a particular good entering the market. In a flow equilibrium, the buyer and seller continuation values, which are the implicit prices, equate flow supply to flow demand for each good that is traded in the market. The competitive equilibrium allocations for economy $I=$ $B \cup S$ is described by the following linear program (and its dual) which is the classical Assignment Problem where fractional assignments are permitted. This formulation is a generalization of Shapley and Shubik (1972) to a setting where buyers can purchase multiple commodities as in Kelso and Crawford (1982) or Gul and Stacchetti (1999, 2000).

\section{Primal}

$$
P=\max _{q \geq 0} \sum_{b \in B} \sum_{G \subset S} q_{b G}\left(h_{b G}-\sum_{s \in G} r_{s}\right)
$$

Subject to

$$
\begin{array}{r}
\sum_{b \in B} \sum_{s \ni G} q_{b G} \leq 1 \text { for all } s \\
\sum_{G \subset S} q_{b G} \leq 1 \text { for all } b .
\end{array}
$$

The vector $q$ that solves the program is a competitive allocation and denotes the measure of matches between buyer $b$ and sellers in the set $G$ that are created in each period of time. Any vector $v$ that solves the dual program is a competitive equilibrium utility vector and the competitive price of a traded good is $p_{x_{s}}=v_{s}+r_{s}$. The constraint given by equation (2) states that the flow demand for seller of type $s$, i.e., $\sum_{b \in B} \sum_{s \ni G} q_{b G}$, must be less than the flow supply of that type, which is at most one. This constraint will bind, if the good's price is positive, or more precisely, if $v_{s}>0$ and thus $p_{x_{s}}=v_{s}+r_{s}>r_{s}$. The constraint given by equation (3) states that the flow supply to buyers of type $b$, must be less than the flow demand by type $b$, which is at most one. Again, this constraint will bind if $v_{b}>0$. Together inequalities (2) and (3) ensure market clearing.

\section{Convergence to Competitive Equilibria}

The following shows that a steady state search equilibrium exists for any configuration of search frictions, that is, for any $\delta$ and $c$ (Theorem 2 and Corollary 2). Also, as search becomes costless, i.e., $\delta \rightarrow 1$ and $c \rightarrow 0$, any sequence of steady state equilibria converges to a competitive equilibrium (Theorem 1 and Corollary 1). The analysis focuses on sequences of equilibria $\left(l^{n}, \sigma^{n}\right)$, and the associated sequences of equilibrium match probabilities $m^{n}$, type distributions $p^{n}$ and values $v^{n}$, as search costs disappear, i.e., as $\left(c^{n}, \delta^{n}\right) \rightarrow(0,1)$.

Let

$$
q_{b(i) G(i)}^{n}=l_{i} \sigma_{i}(\text { out })+\left(1-\sigma_{i}^{n}(\text { in })\right) \sum_{s \in G(i)} l_{i \backslash s} M_{i \backslash s s}
$$


denote measure of buyers with initial type $b(i) \in B$ leaving the market with bundle $G(i)$. Since the market is in steady state $0 \leq q_{b G}^{n} \leq 1$ for all $b \in B, G \subset S$ and $n$. Also, let

$$
e_{b G}^{n}=h_{b G}-\sum_{s \in G} r_{s}-v_{b}-\sum_{s \in G} v_{s}
$$

denote the Excess between any initial buyer type $b \in B$ and sellers in the set $G$; and similarly

$$
e_{i s}=\delta v_{i \cup s}-\delta v_{i}-\delta v_{s}-r_{s}
$$

denote the excess between buyer $i \in T$ and seller $s$. Note that, $0 \leq v_{i}^{n} \leq \bar{h}$; and $-(|S|+1)(\bar{h}+\bar{r}) \leq$ $e_{i j}^{n} \leq \bar{h}$. Consequently, the sequence $\left(q^{n}, e^{n}, v^{n}, p^{n}\right)$ is included in a compact set and has a convergent subsequence. From hereon restrict attention to convergent subsequences $\left(q^{n}, e^{n}, v^{n}, p^{n}\right) \rightarrow$ $(\hat{q}, \hat{s}, \hat{e}, \hat{p})$.

Assume that the explicit search $\operatorname{costs} c^{n}$ do not converge to zero faster than the implicit time $\operatorname{costs} \delta^{n}$. This assumption is stated more precisely below. This assumption ensures that the market is not clogged up by agents, that have no hope of trading and no incentive to leave, accumulating in the economy.

Assumption: Uniform Rate of Convergence (URC). There exists an $r$ such that $r c^{n} \geq\left(1-\delta^{n}\right)$ for all $n$.

Initially assume that in the first period for each agent, the agent does not pay the search cost $c$, that is, the first draw from the distribution comes for free. This assumption ensures that all agents enter the market and there are no coordination problems in entry that could result in a missing market 11 At the end of this subsection, this assumption that the first draw comes for free is relaxed. Also, since the first draw is free, $1 \leq l_{b}$, for all $b$ and $1 \leq l_{s}$ for all $s$.

Assumption: Free First Draw (FD). The search cost $c$ is not paid in the first period for an agent and a unit measure of each type enters the market in each period. Consequently, all agents sample the distribution at least once.

Formally the assumption requires that the choice of not-entering the market and opting for an outside option is not available to agents at the start of their first period in the market. This choice becomes available only after one period in the market.

The following theorem shows that any sequence of steady state search equilibria converges to a competitive equilibrium under the (URC) and (FD) assumptions. The proof first shows that the per-period exit rate of buyers with goods in the set $G$ (i.e., $\hat{q}_{b G}$ ) is a feasible choice for the Assignment Problem and so the flow creation of value in the economy is at most as large as the maximized value of the Assignment Problem. The argument proceeds to show that the Excess $\hat{e}_{i j}$ between any buyer $i$ and seller $j$ as well as the Excess between any initial type $b$ and sellers in the

\footnotetext{
${ }_{1}$ If the first draw was not for free, then no agent entering the economy is an equilibrium. Also, see Example 1 at the end of the section for a more robust coordination failure.
} 
set $G$, i.e., $\hat{e}_{b G}$ is non-positive. No Excess then implies that the vector of equilibrium values $v$ is a feasible choice for the dual of the Assignment Problem and consequently that the flow creation of value in the economy is at least as large as the maximized value of the Assignment Problem. The (URC) and (FD) assumptions together essentially imply that if a buyer waits long enough, then she can meet any seller and make this seller a take-it-or-leave-it offer. This drives the Excess between any two agents to zero as search frictions vanish.

Theorem 1. Assume (URC) and (FD). If $\left(q^{n}, v^{n}\right) \rightarrow(\hat{q}, \hat{v})$, then $\hat{q}$ solves the primal Assignment Problem and is a competitive equilibrium allocation; $\hat{v}$ solves the dual Assignment Problem and is a competitive equilibrium utility vector; and $\hat{v}_{s}+r_{s}$ is a competitive equilibrium price for good $x_{s}$.

Proof. Note that $\sum_{G \subset S} l_{b_{G}}^{n}$ is the measure of buyers, whose initial type was $b$, present in the market. $\sum_{G \subset S} l_{b_{G}}^{n}$ is in steady state since it is the sum of the steady state measures $l_{b_{G}}^{n}$. The number of buyers, whose initial type was $b$, permanently leaving the market in each period is $\sum_{G \subset S} q_{b G}^{n}$ and the number entering is 1 . Consequently, steady state implies $\sum_{G} q_{b G}^{n} \leq \sigma_{b}^{n}(i n)=1$. Also, note that $\sum_{b} \sum_{s \ni G} l_{b_{G}}^{n}+l_{s}^{n}$ denotes the measure of agents who own the good that initially belonged to a seller of type $s$ and this measure is also in steady state since it is just a sum of steady state variables. In each period, the measure of agents leaving with a good that initially belonged to a seller of type $s$ is

$$
\sum_{b} \sum_{s \ni G} q_{b G}^{n}+l_{s}^{n} \sigma_{s}^{n}(\text { out })
$$

and the number of type $s$ agents entering the market is $\sigma_{s}(i n)$. Consequently,

$$
\sum_{b} \sum_{s \ni G} q_{b G}^{n}+l_{s}^{n} \sigma_{s}^{n}(\text { out })=1 .
$$

Taking limits shows

$$
\begin{gathered}
\sum_{b} \sum_{s \ni G} \hat{q}_{b G} \leq 1 \text { for all } s \text { and } \\
\sum_{G} \hat{q}_{b G} \leq 1 \text { for all } b .
\end{gathered}
$$

This implies that the vector $\hat{q}$ satisfies equation (2) and equation (3) and is feasible for the primal Assignment Problem. Consequently,

$$
\sum_{b \in B} \sum_{G \subset S} \hat{q}_{b G}\left(h_{b G}-\sum_{s \in G} r_{s}\right) \leq P .
$$

By Lemma 4 in the appendix, $\hat{e}_{b G} \leq 0$ for all $b$ and $G$, this implies that $\hat{v}$ is feasible for the dual and consequently, $\sum_{B} \hat{v}_{b}+\sum_{S} \hat{v}_{s} \geq D$. But,

$$
\sum_{B} \hat{v}_{b}+\sum_{S} \hat{v}_{s} \leq \sum_{b \in B} \sum_{G \subset S} \hat{q}_{b G}\left(h_{b G}-\sum_{s \in G} r_{s}\right)
$$


by Lemma 5 in the appendix. Consequently,

$$
D \leq \sum_{B} \hat{v}_{b}+\sum_{S} \hat{v}_{s}=\sum_{b \in B} \sum_{G \subset S} \hat{q}_{b G}\left(h_{b G}-\sum_{s \in G} r_{s}\right) \leq P=D
$$

and so $\sum_{B \times S} \hat{q}_{b s} f_{b s}=P$ proving that $\hat{q}$ is a competitive allocation and $\hat{v}$ is a competitive equilibrium utility vector.

Below that assumption that all agents receive their first draw for free is dropped and replaced by a "tighter" version. In particular, following provides a necessary and sufficient condition for every sequence of search equilibria to convergence to a competitive equilibrium. The condition requires that an arbitrarily small but positive measure of the lowest cost seller of each good receive their first draw for free (or enter the market by accident).

Assumption: FD for Low Cost Sellers (FDL). In each period, there is $\varepsilon_{x}>0$ entry by the lowest cost seller of each good $x$.

Again, formally this assumption requires that the choice of not-entering the market and opting for an outside option is not available for a fraction $\varepsilon_{x}>0$ of sellers at the start of their first period in the market. This choice becomes available only after one period in the market.

The following corollary establishes convergence to competitive equilibrium under this assumption. The argument shows that (FDL) and (URC) together are sufficient to show No Excess between any two agents.

Corollary 1. Assume (URC) and (FDL). If $\left(q^{n}, v^{n}\right) \rightarrow(\hat{q}, \hat{v})$, then $\hat{q}$ solves the primal Assignment Problem and is a competitive equilibrium allocation; $\hat{v}$ solves the dual Assignment Problem and is a competitive equilibrium utility vector; and $\hat{v}_{s}+r_{s}$ is a competitive equilibrium price for good $x_{s}$.

Proof. To show convergence, $\hat{e}_{b G} \leq 0$ (no excess) is established. Once, $\hat{e}_{b G} \leq 0$, then the corollary follows from the argument in Theorem 1. Lemma 2 and Lemma 3 are valid under the assumption of the corollary. Also, Lemma 4 can also be applied as follows: Let $i_{x}$ denote the lowest cost seller of good $x$. By Lemma $4, \hat{e}_{G b} \leq 0$ for all $b$ and $G \subset\left\{i_{x}\right\}$.

For any two sellers of good $x, v_{s}-v_{s^{\prime}} \leq v_{s}(i n)-v_{s^{\prime}}(i n)$ and so,

$$
\begin{aligned}
\left(v_{s}(i n)-v_{s^{\prime}}(i n)\right)(1-\delta) \leq \beta \sum_{b \in T} p_{b} m_{b s}\left(r_{s^{\prime}}+\delta v_{s^{\prime}}-r_{s}-\delta v_{s}\right) & \\
& +(1-\beta) \sum_{b \in T} p_{b} m_{s b}\left(r_{s^{\prime}}+\delta v_{s^{\prime}}-r_{s}-\delta v_{s}\right)
\end{aligned}
$$

Also, suppose, without loss of generality, that $r_{s^{\prime}} \geq r_{s}$.

$$
\begin{aligned}
\left(v_{s}-v_{s^{\prime}}\right)(1-\delta) & \leq\left(\left(r_{s^{\prime}}-r_{s}\right)-\delta\left(v_{s}-v_{s^{\prime}}\right)\right) \sum_{b \in T} M_{s b} \\
\left(v_{s}-v_{s^{\prime}}\right) & \leq\left(r_{s^{\prime}}-r_{s}\right) \frac{\sum_{b \in T} M_{s b}}{(1-\delta)+\delta \sum_{b \in T} M_{s b}} \leq\left(r_{s^{\prime}}-r_{s}\right)
\end{aligned}
$$


Consequently, $\delta v_{s}+r_{s} \leq \delta v_{s^{\prime}}+r_{s^{\prime}}$. For any set $\mathrm{G}$ of sellers, let $H$ denote the set of sellers where each $s \in G$ is replaced by $i_{x_{s}}$, i.e., the lowest cost seller who owns the same good as seller $s$. So, $h_{b G}=h_{b H}$, also, $\delta v_{s}+r_{s} \leq \delta v_{s^{\prime}}+r_{s^{\prime}}$ for any $s^{\prime} \in G$ and $s \in H$ with $x_{s^{\prime}}=x_{s}$. Consequently,

$$
e_{b G}=h_{b G}-\sum_{s \in G}\left(\delta v_{s}+r_{s}\right)-\delta v_{b} \leq h_{b H}-\sum_{s \in H}\left(\delta v_{s}+r_{s}\right)-\delta v_{b}=e_{b H}
$$

However, $e_{b H}^{n} \rightarrow \hat{e}_{b H} \leq 0$ since $H \subset i_{x}$. So, $\hat{e}_{b G}=\lim e_{b G}^{n} \leq \lim e_{b H}^{n} \leq 0$ proving that $\hat{e}_{b G} \leq 0$.

As pointed out the condition outlined in Assumption (FDL) is also necessary in the following limited sense: if Assumption (FDL) does not hold, then there exists a sequence of steady state equilibria for an economy that fails to converge to a competitive equilibrium of that economy. The following is such an example.

Example: Necessity of FDL. Consider an economy with two buyer types and two seller types, where each buyer wants to purchase only one good and the two seller types own two different goods. Let $h_{12}=h_{21}=0$ and $h_{11}=h_{22}=1$, that is $h$ is super-modular; buyer 1 likes seller 1 's good and buyer 2 likes seller 2's good. Suppose $r_{1}=r_{2}=0$. Let $\delta=1$. For any $c \leq 1 / 2$, a unit measure of type 1 buyers and a unit measure of type 1 sellers entering, no type 2 buyers or sellers entering and all meetings resulting in a trade at a price of $1 / 2$ is an equilibrium. Clearly such a sequence does not converge to the competitive equilibrium of the economy. However, if a tiny fraction $\varepsilon_{2}$ of type 2 sellers where to enter in each period, then for $c \leq \frac{\varepsilon_{2}}{1+\varepsilon_{2}}$ the buyers of type 2 would also find it profitable to enter. This results in the markets for both goods operating and leads to convergence to a competitive equilibrium.

3.1. Existence of a Steady State Search Equilibrium. The following theorem establishes equilibrium existence for any $\delta \in[0,1]$ and $c>0$. A trivial no-trade equilibrium always exists. The theorem below assumes (FD) (or (FDL)) and consequently establishes the existence of a full-trade equilibrium.

Theorem 2. Assume $(F D)$ or $(F D L)$. For any $(c, \delta)$ a search equilibrium $(l, \sigma)$ exists.

Assumption: Uniqueness (UNQ). The set of goods traded in any competitive equilibrium is the same. That is if good $x$ is not traded in one competitive equilibrium, then it is not traded in any other CE.

Assumption: Decreasing Returns (DR). If $G \subset H$, then $h_{b H \cup\{s\}}-h_{b H} \leq h_{b G \cup\{s\}}-h_{b G}$ for all $b$ and $s$.

Corollary 2. Assume (URC), (UNQ) and (DR). There exists a sequence $\left(q^{n}, v^{n}\right) \rightarrow(\hat{q}, \hat{v})$, such that $\hat{q}$ solves the primal Assignment Problem and is a competitive equilibrium allocation; $\hat{v}$ solves the dual Assignment Problem and is a competitive equilibrium utility vector; and $\hat{v}_{s}+r_{s}$ is a competitive equilibrium price for good $x_{s}$. 
Proof. By (UNQ), the set of goods can be partitioned into two sets $H \subset X$ and $X \backslash H$ where $H$ denotes the set of goods that are traded in any competitive equilibrium. Let $q_{x}$ denote the measure of good $x$ traded by the lowest cost sellers of good $x$, i.e., by sellers $S_{\underline{\mathrm{X}}}=\left\{s: x_{s}=x\right.$ and $r_{s} \leq$ $r_{s}^{\prime}$ for all $s^{\prime}$ with $\left.x_{s^{\prime}}=x\right\}$, traded in a competitive equilibrium. More precisely

$$
q_{x}=\sum_{s \in S_{\underline{\mathbf{X}}}} \sum_{b} \sum_{s \ni G} q_{b G}
$$

Also, let $\underline{q}_{x}=\min _{q \in Q} q_{x}$ where $Q$ denotes the set of competitive allocations. Note that $Q$ is a compact and convex set and $\underline{q}_{x}>0$ for any $x \in H$. Assume (FDL) for all $x \in H$ and let the measure of low cost sellers of good $x \in H$ receiving the first draw free be $0<\varepsilon_{x}<\underline{q}_{x}$. Observe that given this set-up, the sequence of equilibria will converge to $\hat{q}$, which is competitive equilibrium for the economy comprised of sellers such that $x_{s} \in H$ and $b \in B$. Also, observe that since only goods in $H$ are traded, $q$ is also an efficient allocation for the original economy $I$. For any buyer $b$ with $\hat{l_{b}}>0, \hat{e}_{b G} \leq 0$ for any $G \subset S$. For any buyer with $\hat{l_{b}}=0, \hat{e}_{b G} \leq 0$ for any $G \subset\left\{s: x_{s} \in X \backslash H\right\}$. This is because otherwise, i.e., is $\hat{e}_{b G}>0$, then allocating to $b$, who is not trading, the goods in $G$, which are not being traded, would improve the efficiency of the matching which would contradict that the matching $\hat{q}$ is efficient. So $\hat{e}_{b G} \leq 0$ for $G \subset\left\{s: x_{s} \in X \backslash H\right\}$. Also, for $\hat{l}_{b}=0, \hat{e}_{b G} \leq 0$ for any $G \subset\left\{s: x_{s} \in H\right\}$ by Corollary 1. But, $\hat{e}_{b G} \leq 0$ for $G \subset\left\{s: x_{s} \in H\right\}$ and $G \subset\left\{s: x_{s} \in X \backslash H\right\}$ in conjunction with (DR) implies that $\hat{e}_{b G} \leq 0$ for all $b$ and $G \subset S$. This, in turn, shows that the allocation $\hat{q}$ is a competitive equilibrium allocation for $I$ and $\hat{v}$ is a competitive utility vector.

Now observe that for sufficiently large $n, \sigma_{s_{x}}^{n}(\mathrm{in})>\varepsilon_{x}$ since the measure of lowest cost sellers leaving the market must converge to competitive competitive equilibrium which exceeds $\underline{q}_{x}$. This implies that for $n$ sufficiently large $v_{s}(i n) \geq 0$. This shows that we can drop the (FDL) assumption which is not binding for sufficiently large $n$ and just take entry by type $s_{x}$ to equal $\sigma_{s_{x}}^{n}(i n)$.

\section{Discussion And Conclusion}

\section{Appendix A. Omitted Proofs}

A.1. Proof of Lemma 1. Proof of item (i) is bellow. Item (ii) follows from Riley and Zeckhauser (1983). Item (iii) follows since Corollary 1 showed that $\delta v_{s}+r_{s} \leq \delta v_{s^{\prime}}+r_{s^{\prime}}$ for two sellers of the same good with $r_{s} \leq r_{s^{\prime}}$. A similar argument in the non-stationary case yields $\delta v_{s}+r_{s} \leq \delta v_{s^{\prime}}+r_{s^{\prime}}$. The take-it-or-leave-it offer can be viewed as the choice of an optimal monopoly price where marginal cost is equal to $\delta v_{s}+r_{s}$. Consequently, if $\delta v_{s}+r_{s} \leq \delta v_{s^{\prime}}+r_{s^{\prime}}$, then the optimal monopoly price $t_{s} \leq t_{s^{\prime}}$.

I show, if $\mu_{i}=\left(m_{i}, t_{i}\right)$ is the optimal mechanism for type $i$, when type $i$ is proposing and her type is known to the responder, then the mechanism $\mu=\left(\mu_{i}\right)$ is an optimal mechanism choice for all $i$, when the proposer's type is private information. The proof follows the line of reasoning in Yilankaya (1999). By the Inscrutability Principal of Myerson (1983), we can assume without loss of generality, that all proposers choose the same mechanism. This mechanism choice by the proposer 
cannot do better in expectation than the ex-ante optimal mechanism. Below it is shown that the exante optimal mechanism is just $\mu=\left(\mu_{i}\right)$. However, the mechanism choice $\mu=\left(\mu_{i}\right)$ is also available for the proposer and will therefore be chosen. Let $\hat{r}_{s}=r_{s}+\delta v_{s} \geq 0$ and $\hat{h}_{b_{G}}=\delta\left(v_{b_{G \cup s}}-v_{b_{G}}\right)$. The ex-ante problem for the proposer, if the proposer is a seller of good $x$, is as follows:

$$
\max _{m, t \geq 0} \sum_{B_{\mathcal{P}(G)} \times S} p_{b} p_{s}\left(t_{b, s}-m_{b s} \hat{r}_{s}\right)
$$

Subject to the incentive compatibility constraint

$$
\sum_{S} p_{s}\left(m_{j s} \hat{h}_{b}-t_{j s}\right) \leq \sum_{S} p_{s}\left(m_{b, s} \hat{h}_{b}-t_{b, s}\right)
$$

the individual rationality constraint

$$
\sum_{S} p_{s}\left(m_{b, s} \hat{h}_{b}-t_{b, s}\right) \geq 0
$$

and the resource constraint $m_{b s} \leq 1$. Alter the problem as follows: let $h_{b}=\max \left\{\hat{h}_{b}, 0\right\}$ for all $b \in B_{\mathcal{P}(G)}$ and add a buyer 0 with $h_{0}<0$ and $p_{0}=0$. Also, drop the individual rationality constraint for all buyers except buyer 0 . The modified ex-ante problem is then

$$
\begin{array}{rlr}
V^{x}=\max _{m, t \geq 0} & \sum_{B_{\mathcal{P}(G)} \times S} p_{b} p_{s}\left(t_{b s}-m_{b, s} \hat{r}_{s}\right) & \\
\sum_{S} p_{s}\left(m_{i s} h_{b}-t_{i s}\right) & \leq \sum_{S} p_{s}\left(m_{b, s} h_{b}-t_{b_{G}, s}\right) \forall b \text { and } i & \left(\alpha_{b j}^{x}\right) \\
0 & \leq \sum_{S} p_{s}\left(m_{0 s} h_{b}-t_{0 s}\right) & \left(\psi^{x}\right) \\
m_{b s} & \leq 1
\end{array}
$$

where the set $B$ now includes buyer 0 and the Lagrange multipliers are given to the right. The modified problem and the original problem have the same payoff for the proposer. To see this pick any solution to the original problem and take $m_{0 s}=t_{0 s}=0$. To show that this is feasible for the modified problem we only need to check constraints for agents with $\hat{h}_{b} \leq 0$ and for $h_{0}$. However, for any such agent, their utility in both the original and modified problem is zero which is their maximum attainable utility. Consequently, the solution to the original problem is feasible for the modified problem and the payoff for the modified problem is at least as large as the original problem. Pick any solution to the modified problem and set $m_{b s}=t_{b s}=0$ for any $h_{b} \leq 0$ which must also a solution to the modified problem. To show that this solution is also feasible for the original problem note that for any $h_{b} \leq 0, m_{b s}=0$ and $t_{b s}=0$ and so these agents receive their highest possible utility and both IR and IC constraints for these agents hold. Also, dropping the individual rationality constraints causes no change in value since any agent can still guarantee non-negative payoff by pretending to be agent 0 . Consequently, the value for the original problem must be at least as large as the value for the modified problem showing that the two payoffs are 
equal. The dual of the ex-ante problem is

$$
\begin{gathered}
D^{x}=\min _{\gamma^{x} \geq 0, \alpha \geq 0, \psi \geq 0} \sum_{B \times S} \gamma_{b s}^{x} \\
\sum_{j \in B}\left(h_{b} \alpha_{b j}^{x}-\hat{h}_{j} \alpha_{j b}^{x}\right) \leq \frac{\gamma_{b s}^{x}}{p_{s}}+p_{b} \hat{r}_{s} \forall b \neq 0 \text { and } s, \\
\sum_{j \in B}\left(\alpha_{j b}^{x}-\alpha_{b j}^{x}\right)+p_{b} \leq 0 \forall b \neq 0, \\
\sum_{j \in B}\left(\alpha_{j 0}^{x}-\alpha_{0 j}^{x}\right) \leq \psi^{x}, \\
\sum_{j \in B}\left(h_{0} \alpha_{0 j}^{x}-h_{j} \alpha_{j 0}^{x}\right)+\hat{h}_{0} \psi^{x} \leq \frac{\gamma_{0 s}^{x}}{p_{s}} \forall s .
\end{gathered}
$$

Observe that the last constraint for the dual problem is satisfied automatically and can be ignored since, for any choice of $\gamma^{x} \geq 0, \alpha \geq 0, \psi \geq 0$, the left hand side is always non-positive and the right hand side is always non-negative.

Similarly, the problem when $\hat{r}_{s}$ is know at it's dual are formulated as follows:

$$
V_{s}=\max _{m, t \geq 0} \sum_{B} p_{b}\left(t_{b s}-m_{b s} \hat{r}_{s}\right)
$$

subject to:

$$
\begin{aligned}
h_{b} m_{j s}-t_{j s} & \leq h_{b} m_{b s}-t_{b s} b \text { and } j \\
0 & \leq h_{0} m_{0 s}-t_{0 s} \\
m_{b s} & \leq 1
\end{aligned}
$$

and the dual is,

$$
D_{s}=\min _{\gamma \geq 0} \sum_{b} \gamma_{b s}
$$

subject to:

$$
\begin{aligned}
\sum_{j \in B}\left(h_{b} \alpha_{b j}^{s}-\hat{h}_{j} \alpha_{j b}^{s}\right) & \leq p_{b} \hat{r}_{s}+\gamma_{b s} \forall b \neq 0, \\
\sum_{j \in B}\left(\alpha_{j b}^{s}-\alpha_{b j}^{s}\right)+p_{b} & \leq 0 \forall b \neq 0, \\
\sum_{j \in B}\left(\alpha_{j 0}^{s}-\alpha_{0 j}^{s}\right) & \leq \psi^{s}, \\
\sum_{j \in B}\left(h_{0} \alpha_{0 j}^{s}-h_{j} \alpha_{j 0}^{s}\right)+h_{0} \psi^{s} & \leq \gamma_{0 s} .
\end{aligned}
$$

Again, the last constraint for the dual problem can be ignored.

Let $m_{s}$ and $t_{s}$ solve the mechanism choice problem when the cost is $r_{s}$ is known, and let $\alpha_{s}$ $\gamma_{s}$ and $\psi_{s}$ denote a dual solution. Observe that $m=\left(m_{s}\right)$ and $t=\left(t_{s}\right)$ is a feasible choice for the ex-ante problem since if each choice satisfies $I C$ and $I R_{0}$ separately, they satisfy $I C$ and $I R_{0}$ on average. This implies that $V^{x} \geq \sum_{s} p_{s} V_{s}$. Also, observe that $\alpha=\left(\alpha^{s}\right), \gamma=\left(p_{s} \gamma^{s}\right)$ and 
$\psi=\max _{s}\left\{\psi^{s}\right\}$ is feasible for the dual of the ex-ante problem. Consequently, $D^{x} \leq \sum_{B \times S} p_{s} \gamma_{s b}$. However, $D^{x} \leq \sum_{B \times S} p_{s} \gamma_{s b}=\sum_{s} p_{s} V_{s} \leq V^{x}$ and so $V^{x}=\sum_{s} p_{s} V_{s}$ completing the proof.

\section{A.2. Proof of Theorem 1.}

Lemma 2 (No Excess 1). If $\max \left\{\hat{p}_{b}, \hat{p}_{s}\right\}>0$, then $e_{b s} \leq 0$.

Proof. For any $c^{n}$ and $\delta^{n}$ a seller (or buyer) can offer to sell her good for $\delta^{n} v_{b \cup s}-\delta^{n} v_{b}-\varepsilon$ and ensure that buyer $b$ purchases if they meet, since the payoff that buyer $b$ gets from purchasing the good strictly exceeds her continuation payoff $\delta^{n} v_{b}$. Also, any buyer can offer to buy a good for $r_{s}+\delta^{n} v_{s}^{n}+\varepsilon$, and ensure that she makes a purchase if she meets seller $s$. Consequently,

$$
\begin{aligned}
v_{s}^{n} & \geq-c^{n}+(1-\beta) p_{b}^{n}\left(\delta^{n} v_{b \cup s}-\delta^{n} v_{b}-r_{s}\right)+\delta^{n}\left(1-(1-\beta) p_{b}^{n}\right) v_{s}^{n} \\
\left(1-\delta^{n}\right) v_{s}^{n} & \geq-c^{n}+(1-\beta) p_{b}^{n}\left(\delta^{n} v_{b \cup s}-\delta^{n} v_{b}-\delta^{n} v_{s}^{n}-r_{s}\right) \\
\left(1-\delta^{n}\right) v_{s}^{n} & \geq-c^{n}+(1-\beta) p_{b}^{n} e_{b s}^{n}
\end{aligned}
$$

and

$$
\begin{aligned}
& \left(1-\delta^{n}\right) v_{b}^{n} \geq-c^{n}+\beta p_{s}^{n}\left(\delta^{n} v_{b \cup s}-\delta^{n} v_{b}-\delta^{n} v_{s}^{n}-r_{s}\right), \\
& \left(1-\delta^{n}\right) v_{b}^{n} \geq-c^{n}+\beta p_{s}^{n} e_{b s}^{n}
\end{aligned}
$$

Taking limits shows that $\hat{p}_{b} \hat{e}_{b s} \leq 0$ and $\hat{p}_{s} \hat{e}_{b s} \leq 0$. However, since $\max \left\{\hat{p}_{b}, \hat{p}_{s}\right\}>0, \hat{e}_{b s} \leq 0$.

Lemma 3. Let $L^{n}=\max \left\{L_{B}^{n}, L_{S}^{n}\right\}, \lim _{n} c^{n} L^{n}=0$ and $\lim _{n}\left(1-\delta^{n}\right) L^{n}=0$.

Proof. If $\lim \sup L^{n}<\infty$, then since $0 \leq L^{n}, \lim _{n} c^{n} L^{n}=0$ and $\lim _{n}\left(1-\delta^{n}\right) L^{n}=0$. If $\lim \sup L^{n}=$ $\infty$, then there must exist an agent type $i$ with $\hat{p}_{i}>0$ for whom $l_{i}^{n} \rightarrow \infty$ and so the value from staying in the market for this type agent must be non-negative for all $n$ large. Consequently, if $\limsup L^{n}=\infty$, then there exists a buyer $b$ with $\hat{p}_{b}>0$ and $v_{b}^{n}(i n) \geq 0$, or a seller $s$ with $\hat{p}_{s}>0$ and $v_{s}^{n}(i n) \geq 0$, for all $n$ large. Assume, without loss of generality, that there exists a buyer $b$ with $\hat{p}_{b}>0$ and $v_{b}^{n}(i n) \geq 0$. This implies for sufficiently large $n$,

$$
\begin{aligned}
\left(1-\delta^{n}\right) l_{b}^{n} v_{b}^{n}+l_{b}^{n} c^{n}=\beta \sum_{S} l_{b}^{n} p_{s}^{n} m_{b s}^{n} & \left(\delta^{n} v_{b \cup s}-t_{b s}^{n}-\delta^{n} v_{b}^{n}\right) \\
& +(1-\beta) \sum_{S} l_{b}^{n} p_{s}^{n} m_{b s}^{n}\left(\delta^{n} v_{b \cup s}-t_{s b}^{n}-\delta^{n} v_{b}^{n}\right)
\end{aligned}
$$


However, for $m_{b s}^{n}>0, t_{b s}^{n} \geq \delta^{n} v_{s}^{n}+r_{s}$, so $\delta^{n} v_{b \cup s}-t_{b s}^{n}-\delta^{n} v_{b}^{n} \leq \delta^{n} v_{b \cup s}-\delta^{n} v_{b}-\delta^{n} v_{s}^{n}-r_{s}$ and $\delta^{n} v_{b \cup s}-t_{s b}^{n}-\delta^{n} v_{b}^{n} \leq \delta^{n} v_{b \cup s}-\delta^{n} v_{b}-\delta^{n} v_{s}^{n}-r_{s}$. Consequently,

$$
\begin{aligned}
\left(1-\delta^{n}\right) l_{b}^{n} v_{b}^{n}+l_{b}^{n} c^{n} & \leq \beta \sum_{S} l_{b}^{n} p_{s}^{n} m_{b s}^{n} e_{b s}^{n}+(1-\beta) \sum_{S} l_{b}^{n} p_{s}^{n} m_{b s}^{n} e_{b s}^{n} \\
\left(1-\delta^{n}\right) l_{b}^{n} v_{b}^{n}+l_{b}^{n} c^{n} & \leq \bar{e}_{b s}^{n} \sum_{S} l_{b}^{n} M_{b s}^{n} \leq \bar{e}_{b s}^{n} \\
\frac{1}{p_{b}^{n}}\left(\left(1-\delta^{n}\right) l_{b}^{n} v_{b}^{n}+l_{b}^{n} c^{n}\right) & \leq \frac{\bar{e}_{b s}^{n}}{p_{b}^{n}}
\end{aligned}
$$

However, since $\hat{p}_{b}>0$ by Lemma $2 \lim _{n} e_{b j}^{n}=0$ for any $j$. This implies that

$$
\begin{aligned}
\lim _{n} \frac{1}{p_{b}^{n}}\left(\left(1-\delta^{n}\right) l_{b}^{n} v_{b}^{n}+l_{b}^{n} c^{n}\right) & \leq \lim _{n} \frac{\bar{e}_{b s}^{n}}{p_{b}^{n}} \\
\lim _{n}\left(1-\delta^{n}\right) L^{n} v_{b}^{n}+L^{n} c^{n} & =0
\end{aligned}
$$

Observe since $\lim L^{n} c^{n}=0$, by Assumption $1, \lim _{n}\left(1-\delta^{n}\right) L^{n}=0$.

Lemma 4. If $\hat{p}_{b}=0$, then also $\hat{e}_{b G} \leq 0$, consequently, $\hat{e}_{b G} \leq 0$ for all $b$ and $G$.

Proof. By the argument provided in Lemma 2

$$
\left(1-\delta^{n}\right) v_{b}^{n} \geq-c^{n}+\beta p_{s 1}^{n}\left(\delta^{n} v_{b_{\{s 1\}}}-\delta^{n} v_{b}^{n}-\delta^{n} v_{s 1}^{n}-r_{s 1}^{n}\right) .
$$

Multiply both sides by $L^{n}=\max \left\{L_{B}^{n}, L_{S}^{n}\right\}$ which gives

$$
\left(\left(1-\delta^{n}\right) v_{b}^{n}+c^{n}\right) L^{n} \geq \beta L^{n} p_{s 1}^{n}\left(\delta^{n} v_{b_{\{s 1\}}}^{n}-\delta^{n} v_{b}^{n}-\delta^{n} v_{s 1}^{n}-r_{s 1}^{n}\right) .
$$

Note that $L^{n} p_{s}^{n}=l_{s}^{n} \geq 1$ for all $n$. However, by Lemma 3

$$
\lim _{n}\left(\left(1-\delta^{n}\right) v_{b}^{n}+c^{n}\right) L^{n}=0 .
$$

Consequently,

$$
\hat{v}_{b_{\{s 1\}}}-\hat{v}_{b}-\hat{v}_{s 1}-r_{s 1} \leq 0 .
$$

Also, again by the argument provided in Lemma 2 ,

$$
\left(\left(1-\delta^{n}\right) v_{b_{\{s 2\}}}^{n}+c^{n}\right) L^{n} \geq \beta L^{n} p_{s 2}^{n}\left(\delta^{n} v_{b_{\left\{s_{1}, s 2\right\}}}-\delta^{n} v_{b\left\{s_{1}\right\}}^{n}-\delta^{n} v_{s 2}^{n}-r_{s 2}^{n}\right) .
$$

So, $\hat{v}_{b_{\left\{s_{1}, s 2\right\}}}-\hat{v}_{b_{\left\{s_{1}\right\}}}-\hat{v}_{s 2}-r_{s 2} \leq 0$. Substituting gives

$$
\hat{v}_{b_{\left\{s_{1}, s 2\right\}}}-\hat{v}_{b}-\hat{v}_{s 2}-\hat{v}_{s 1}-r_{s 1}-r_{s 2} \leq 0
$$

Repeating $|G|$ times shows that

$$
\hat{v}_{b_{G}}-\hat{v}_{b}-\sum_{s \in G}\left(\hat{v}_{s}+r_{s}\right) \leq 0 .
$$

However, $v_{b_{G}}^{n} \geq h_{b G}$ for all $n$ and so $\hat{v}_{b_{G}} \geq h_{b G}$. Thus

$$
h_{b G}-\hat{v}_{b}-\sum_{s \in G}\left(\hat{v}_{s}+r_{s}\right) \leq 0
$$


proving the result.

Lemma 5. $\sum_{B} \hat{v}_{b}+\sum_{S} \hat{v}_{s} \leq \sum_{b} \sum_{G} \hat{q}_{b G}\left(h_{b G}-\sum_{s \in G} r_{s}\right)$.

Proof. The value equations for the buyers implies

$$
\begin{aligned}
l_{b} v_{b}(1-\delta) \leq \beta \sum_{s} l_{b} p_{s} m_{b s}\left(\sigma_{b \cup s}(i n) \delta\left(v_{b \cup s}-h_{i_{b} G(b \cup s)}\right)+\delta h_{i_{b} G(b \cup s)}-\delta v_{b}-t_{b s}\right) \\
+(1-\beta) \sum_{s} l_{b} p_{s} m_{s b}\left(\sigma_{b \cup s}(i n) \delta\left(v_{b \cup s}-h_{i_{b} G(b \cup s)}\right)+\delta h_{i_{b} G(b \cup s)}-\delta v_{b}-t_{s b}\right)
\end{aligned}
$$

Summing up over all buyers and taking the limit as $\delta \rightarrow 1$ and observing that $t_{b s}$ goes to $v_{s}+r_{s}$ for any $b$ and $s$ with $m_{b s}>0$ gives

$0 \leq \sum_{b_{G} \in T} \sum_{s \in S}\left(1-\hat{\sigma}_{b_{G \cup s}}(i n)\right)\left(\beta \hat{l}_{b_{G}} \hat{p}_{s} \hat{m}_{b_{G} s}+(1-\beta) \hat{l}_{b_{G}} \hat{p}_{s} \hat{m}_{s b_{G}}\right)\left(h_{b G \cup s}-\hat{v}_{b}-\sum_{j \in G \cup s}\left(\hat{v}_{j}+r_{j}\right)\right)$ rearranging shows that

$0 \leq \sum_{b_{G} \in T} \sum_{s \in S}\left(1-\hat{\sigma}_{b_{G}}(i n)\right)\left(\beta \hat{l}_{b_{G \backslash s}} \hat{p}_{s} \hat{m}_{b_{G \backslash s} s}+(1-\beta) \hat{l}_{b_{G \backslash s}} \hat{p}_{s} \hat{m}_{s b_{G \backslash s}}\right)\left(h_{b G}-\hat{v}_{b}-\sum_{j \in G}\left(\hat{v}_{j}+r_{j}\right)\right)$

However

which implies that

$$
\sum_{s \in S}\left(1-\hat{\sigma}_{b_{G}}(i n)\right)\left(\beta \hat{l}_{b_{G \backslash s}} \hat{p}_{s} \hat{m}_{b_{G \backslash s} s}+(1-\beta) \hat{l}_{b_{G \backslash s}} \hat{p}_{s} \hat{m}_{s b_{G \backslash s}}\right)=\hat{q}_{b G}
$$

$$
\begin{aligned}
0 & \leq \sum_{b_{G} \in T} \hat{q}_{b G}\left(h_{b G}-\hat{v}_{b}-\sum_{j \in G}\left(\hat{v}_{j}+r_{j}\right)\right) \\
0 & \leq \sum_{b} \sum_{G} \hat{q}_{b G}\left(h_{b G}-\hat{v}_{b}-\sum_{j \in G}\left(\hat{v}_{j}+r_{j}\right)\right)
\end{aligned}
$$

Observe that for $b$ with $\hat{v}_{b}>0 \sum_{b} \sum_{G} \hat{q}_{b G}=1$ and for $s$ with $\hat{v}_{s}>0, \sum_{b} \sum_{s \ni G} \hat{q}_{b G}=1$ so

$$
\sum_{B} \hat{v}_{b}+\sum_{S} \hat{v}_{s} \leq \sum_{b} \sum_{G} \hat{q}_{b G}\left(h_{b G}-\sum_{s \in G} r_{s}\right)
$$

proving the result.

A.3. Proof of Theorem 2. For any $c$ and $\delta, 0 \leq v_{i} \leq \bar{h}$. Let $V=\left\{v \in R^{|B| \times 2^{|S|}+|S|}: 0 \leq v_{i} \leq \bar{h}\right\}$ denote the set of possible values. The steady state measure for any agent $i$ is bounded. For all $b$ and $s, v_{s}($ in $)<0$. then $l_{s}=1$ and $v_{b}($ in $)<0$, then $l_{b}=1$, by Assumption (FD). If $v_{s}($ in $) \geq 0$, then $l_{s}=1 /\left(\sum_{b \in T} M_{s b}+\sigma_{s}(\right.$ out $\left.)\right)$. If $v_{b}($ in $) \geq 0$, then $l_{b}=1 /\left(\sum_{s \in S} M_{b s}+\sigma_{b}(\right.$ out $\left.)\right)$. Also, if $\sigma_{b}($ in $) \sum_{s \in G(b)} l_{b \backslash s} M_{b \backslash s s}$, then

$$
l_{b}=\frac{\sigma_{b}(\text { in }) \sum_{s \in G(b)} l_{b \backslash s} M_{b \backslash s s}}{\sum_{s \in S} M_{b s}+\sigma_{b}(\text { out })} .
$$

Observe that if $v_{i}($ in $) \geq 0$, then $v_{i}($ in $) \leq-c+\sum_{j} M_{i j} \bar{h}$ and so $c / \bar{h} \leq \sum_{j} M_{i j}$. Consequently, $1 \leq l_{i} \leq \frac{\bar{h}}{c}$. Let $\Lambda=\left\{l: 1 \leq l_{i} \leq \frac{\bar{h}}{c}\right\}$ denote the set of possible steady state measures. Let $m_{b s}$ and 
transfer $t_{b s}$ be the mechanism choice by the buyers and $m_{s b}$ and $t_{s b}$ the mechanism choice by the sellers, and $\sigma_{i}=\left(\sigma_{i 0}, \mu_{i}\right)$. Start with any $l \in \Lambda, \sigma \in \Sigma, v \in V$ and let

$$
\begin{aligned}
& l_{s}^{\prime}(l, \sigma, v)=\frac{1}{\max \left\{c / \bar{h}, \sum_{j \in T} M_{j s} p_{j}\right\}}, \\
& l_{b}^{\prime}(l, \sigma, v)=\frac{1}{\max \left\{c / \bar{h}, \sum_{s \in S} M_{b s}\right\}} \text { and, } \\
& l_{j}^{\prime}(l, \sigma, v)=\frac{\sigma_{j}(i n) \sum_{s \in G(j)} l_{j \backslash s} M_{j \backslash s s}}{\max \left\{c / \bar{h}, \sum_{s \in S} M_{j s}\right\}}
\end{aligned}
$$

where the $M$ 's are calculated according to $\sigma, p$ and $l$. This defines a continuous function from $\Lambda \times \Sigma \times V$ into $\Lambda$, where $(l, \sigma, v) \mapsto l_{i}^{\prime}$ for each $i$.

Let

$$
\begin{aligned}
v_{b}^{\prime}(i n \mid l, \sigma, v) & =\max _{\left(m_{b}^{\prime}, t_{b}^{\prime}\right) \geq 0}-c+\beta \sum p_{s}\left(m_{b s}^{\prime}\left(\delta v_{b \cup s}-\delta v_{b}\right)-t_{b s}^{\prime}\right) \\
& +(1-\beta) \sum_{s} p_{s}\left(m_{s b}\left(\delta v_{b \cup s}-\delta v_{b}\right)-t_{s b}\right)+\delta v_{b}
\end{aligned}
$$

subject to

$$
\begin{aligned}
t_{b s}^{\prime}-m_{b s}^{\prime}\left(r_{s}+\delta v_{s}\right) & \geq t_{b j}^{\prime}-m_{b j}^{\prime}\left(r_{s}-\delta v_{s}\right) \text { for all } s \text { and } j \in S \\
t_{b s}^{\prime}-m_{b s}^{\prime}\left(r_{s}+\delta v_{s}\right) & \geq 0 \text { for all } s \\
m_{b s}^{\prime} & \leq 1 \text { for all } s .
\end{aligned}
$$

Also, let $S_{b, 1}^{\prime}(l, \sigma, v)$ denote the set of maximizers for the above program and

$$
S_{b, 0}^{\prime}(l, \sigma, v)=\arg \max _{\sigma_{0} \in \Delta\{\text { in }, \text { out }\}} \sigma_{0} v_{b}^{\prime}(\text { in }, l, \sigma, v)+\left(1-\sigma_{0}\right) h_{b G(b)} .
$$

Similarly, for a seller, let

$$
\begin{aligned}
v_{s}^{\prime}(i n \mid l, \sigma, v) & =\max _{m_{s}^{\prime}, t_{s}^{\prime}}-c+(1-\beta) \sum_{b \in T} p_{b}\left(t_{s b}^{\prime}-m_{s b}^{\prime}\left(r_{s}+\delta v_{s}\right)\right) \\
& +\beta \sum_{b \in T} p_{b}\left(t_{b s}+m_{b s}\left(r_{s}+\delta v_{s}\right)\right)+\delta v_{s}
\end{aligned}
$$

subject to

$$
\begin{aligned}
m_{s b}^{\prime}\left(\delta v_{b \cup s}-\delta v_{b}\right)-t_{s b}^{\prime} & \geq m_{s j}^{\prime}\left(\delta v_{b \cup s}-\delta v_{b}\right)-t_{s j}^{\prime} \text { for all } b \text { and } j \in T \\
m_{s b}^{\prime}\left(\delta v_{b \cup s}-\delta v_{b}\right)-t_{s b}^{\prime} & \geq 0 \text { for all } b \\
m_{s b}^{\prime} & \leq 1 \text { for all } b
\end{aligned}
$$

Also, let $S_{s, 1}^{\prime}(l, \sigma, v)$ denote the set of maximizers for the above program and

$$
S_{s, 0}^{\prime}(l, \sigma, v)=\arg \max _{\sigma_{0} \in \Delta\{\text { in }, \text { out }\}} \sigma_{0} v_{s}^{\prime}(\text { in }, l, \sigma, v) .
$$


Finally let $S_{i}^{\prime}(l, \sigma, v)=S_{i, 0}^{\prime}(l, \sigma, v) \times S_{s, 1}^{\prime}(l, \sigma, v)$ and $S^{\prime}(l, \sigma, v)=\prod_{i} S_{i}^{\prime}(l, \sigma, v)$. This process defines a continuous function from $\Lambda \times \Sigma \times V$ into $V$ where $(l, \sigma, v) \mapsto v_{i}^{\prime}$ for each $i$ and defines an upper-hemi-continuous, convex compact valued correspondence from $\Lambda \times \Sigma \times V$ into $V$ where $(l, \sigma, v) \mapsto S^{\prime}$, by Berge's Theorem of the Maximum.

However, we have defined an UHC correspondence $(l, \sigma, v) \mapsto\left(l^{\prime}, S^{\prime}, v^{\prime}\right)$. This correspondence maps $\Lambda \times \Sigma \times V$ into $\Lambda \times \Sigma \times V$, it is upper-hemi-continuous, compact, and convex valued; thus by Kakutani's theorem has a fixed point. This fixed point is an equilibrium for the economy.

\section{REFERENCES}

DeFraja, G., AND J. SAkovics (2001): "Walras Retrouve: Decentralized Trading Mechanisms and the Competitive Price," Journal of Political Economy, 109, 842-863.

Gale, D. (1987): "Limit Theorems for Markets with Sequential Bargaining," Journal of Economic Theory, 43, $20-54$. Gul, F., And E. Stacchetti (1999): "Walrasian Equilibrium with Gross Substitutes," Journal of Economic Theory. - (2000): "The English Auction with Differentiated Commodities," Journal of Economic Theory, 92,66 - 95.

Kelso, A., AND V. CRAwford (1982): "Job Matching, Coalition Formation, and Gross Sustitutes," Econometrica, 50(6), 1483-1504.

Lauermann, S. (2006): "Dynamic Matching and Bargaining Games: A general Approach," .

Maskin, E., AND J. Tirole (1990): "The Principal-Agent Relationship with an Informed Principal: The Case of Private Values," Econometrica, 58(2), 379-409.

Moreno, D., And J. Wooders (2002): "Prices, Delay and the Dynamics of Trade," Journal of Economic Theory, $104,304-339$.

Myerson, R. (1983): "Mechanism Design by an Informed Principal," Econometrica, 51(6), 1767-1798.

Riley, J., AND R. Zeckhauser (1983): "Optimal Selling Strategies: When to Haggle, When to Hold Firm," Quarterly Journal of Economics, 98(2), 267-289.

Satterthwaite, M., And A. Shneyerov (2006): "Dynamic Matching, Two-sided Incomplete Information, and Participation Costs: Existence and Convergence to Perfect Competition," Econometrica, Forthcoming.

Serrano, R. (2002): "Decentralized Information and the Walrasian Outcome: A Pairwise Meetings Market with Private Values," Journal of Mathematical Economics, 38, 65-89.

Shapley, L., AND M. Shubik (1972): “The Assignment Game I: The Core," International Journal of Game Theory, $1(2), 111-130$.

Wolinsky, A. (1990): "Information Revelation in a Market Pairwise Meetings," Econometrica, 58, 1-23.

YilankayA, O. (1999): "A Note on the Seller's Optimal Mechanism in Bilateral Trade with Two-Sided Incomplete Information," Journal of Economic Theory, 87, 267-271.

Meds, Kellogg School of Management, Northwestern University, 2001 Sheridan Road, Evanston, IL 60208

E-mail address: a-atakan@kellogg.northwestern.edu 